\title{
Economie de la décision publique et conflits d'usages pour un cadre de vie dans les espaces ruraux et périurbains
}

Philippe Jeanneaux

\section{(2) OpenEdition \\ 12 Journals}

\section{Édition électronique}

URL : http://journals.openedition.org/developpementdurable/2586

DOI : 10.4000/developpementdurable.2586

ISSN : 1772-9971

Éditeur

Association DD\&T

\section{Référence électronique}

Philippe Jeanneaux, « Economie de la décision publique et conflits d'usages pour un cadre de vie dans les espaces ruraux et périurbains », Développement durable et territoires [En ligne], Dossier 7 | 2006, mis en ligne le 10 mai 2006, consulté le 19 avril 2019. URL : http://journals.openedition.org/ developpementdurable/2586 ; DOI : 10.4000/developpementdurable.2586

\section{Ce document a été généré automatiquement le 19 avril 2019.}

\section{(cc) (i) \&}

Développement Durable et Territoires est mis à disposition selon les termes de la licence Creative Commons Attribution - Pas d'Utilisation Commerciale 4.0 International. 


\title{
Economie de la décision publique et conflits d'usages pour un cadre de vie dans les espaces ruraux et périurbains
}

\author{
Philippe Jeanneaux
}

1 Depuis quelques décennies, l'espace rural est de plus en plus recherché pour la qualité de son cadre de vie et il s'affirme comme un lieu de résidence et de récréation pour un nombre croissant de Français, même si les usages productifs restent prégnants. Les ressources de la campagne connaissent de nouvelles concurrences pour leurs usages jusqu'à déboucher parfois sur des oppositions ouvertes communément qualifiées de conflits d'usage. La diversité des oppositions est très variable : ici une association de protection de la nature s'oppose à la création d'un site de stockage de déchets, là un propriétaire foncier conteste le passage de randonneurs sur ses terres, ailleurs encore un maire et ses administrés refusent l'extension d'une porcherie autorisée par un préfet.

2 Dans ce contexte, le travail présenté dans ce papier vise à éclairer le processus d'affectation et de reconnaissance des droits d'usages des ressources localisées du rural qui s'opère lors de la confrontation entre les usages résidentiels, et les usages productifs ou de réservation (non usage). Dans ce cadre, nous considérons que les conflits d'usage combinent une dimension physique et une dimension institutionnelle :

3 - Physique, parce que le conflit d'usage signale la réception par certains agents d'un effet externe technologique négatif, et met en évidence que les préférences des usagers pour les ressources communes localisées constitutives du cadre de vie résidentiel sont incompatibles ;

4 - Institutionnelle, parce que les citoyens, usagers du cadre de vie, prennent la parole pour exprimer leurs prétentions aux autorités publiques, entre deux élections ou faute de procédure du vote. Assimilé dans cette recherche à un processus d'ajustement de la décision publique s'opérant devant les tribunaux et/ou avec le concours de différents moyens de pression médiatique, le conflit permet aux contestataires dans de nombreuses 
situations de modifier la distribution des droits d'usages sur les ressources constitutives de leur cadre de vie en exerçant une pression informelle sur les responsables élus ou nommés chargés d'organiser plus ou moins directement les règles d'usages et/ou la fourniture de biens publics. d'usage pour un cadre de vie. Les concepts de l'économie de la décision publique permettront de traiter la notion de conflit d'usage comme une situation d'ajustement des arrangements institutionnels à l'origine de la distribution des droits d'usage des ressources localisées des espaces ruraux. Dans un deuxième temps, les grilles d'analyse des conflits et la méthodologie seront présentées. Suivront enfin, les premiers résultats et les enseignements tirés de tests réalisés à partir de conflits d'usage observés au Tribunal Administratif et dans un quotidien régional pour le département du Puy de Dôme.

1. Contexte et délimitation économique des conflits d'usage pour un cadre de vie de l'espace rural1.1. L'espace rural en mutation : l'affirmation de la campagne cadre de vie

7 L'analyse des conflits d'usage dans les espaces ruraux et périurbains peut être préalablement nourrie par l'examen des tendances fortes qui se combinent et modifient les rapports entre usagers de l'espace rural (PERRIER-CORNET, 2002a).

8 La montée en puissance de la fonction cadre de vie est la tendance forte qui marque la physionomie des espaces ruraux et périurbains depuis les années 1970. Cette évolution s'explique par des mouvements de migrations des pôles urbains vers les espaces périurbains et ruraux essentiellement motivés par l'usage résidentiel permis par la baisse des coûts de transport et l'augmentation du niveau de vie : "Vivre dans un cadre naturel loin de l'agitation des villes, dans des maisons spacieuses et pourvues de jardins, tout en conservant la source de rémunération qu'est l'emploi en ville, tel est sans nul doute l'objectif que se sont fixé ces familles" (LE JEANNIC, 1997, p. 41). Ces mouvements migratoires sont à l'origine du renouveau démographique de nombreuses zones rurales. Pour CAVAILHES et SCHMITT (2002), certains biens non marchands compris dans la notion d'aménité semblent jouer un rôle non négligeable dans la localisation résidentielle des ménages, même si GOFFETTE NAGOT (1996) n'a pu mettre clairement en évidence le rôle des aménités naturelles dans le choix de localisation des ménages du fait de problèmes de mesures de ces aménités. Toutefois, plus récemment les travaux de LECAT (2004) montrent que l'influence de l'environnement local sur le cadre de vie est intégré dans le marché foncier résidentiel à travers les effets significatifs de différents facteurs tels les sites classés, les chemins de randonnées, ou les bases de plein air. En revanche, le rôle de l'environnement agricole serait difficilement discernable, comme si aucun paysage agricole n'était préféré aux autres.

9 Concernant les autres usages, l'agriculture fait de moins en moins le rural en termes de richesses et d'emplois créés. Dans le même temps, de nombreuses autres activités productives restent soutenues. Les activités industrielles, d'extraction, de stockage, tout comme les infrastructures de transport utilisent très largement les ressources de la campagne et représentent une part importante de l'emploi dans les espaces périurbains et ruraux (AUBERT et BLANC, 2002).

10 Enfin, la conscience écologique s'est affirmée dans des territoires de plus en plus considérés comme des espaces de nature (DEVERRE et al, 2002). La médiatisation des 
atteintes successives portées à l'environnement (pollution, destruction d'espèces, exploitation non contrôlée des ressources naturelles...) et leurs conséquences supposées (changements climatiques, inondations, tempêtes...) a initié l'opinion aux risques de la croissance économique effrénée sous l'influence des courants biocentriste et conservationniste développés depuis les années 1950, et aujourd'hui avec le concept de développement durable (FAUCHEUX et NOEL, 1995).

11 Finalement, les espaces ruraux assurent de nombreuses fonctions (PERRIER-CORNET, 2002a) :

12 - une fonction résidentielle et récréative, caractéristique d'une campagne cadre de vie ;

13 - une fonction de production regroupant les usages agricoles, forestiers, extractifs, de transport, et industriels ;

14 - une fonction de réservation permettant la préservation des biens de nature utiles pour l'homme et les générations futures (conservation de la biodiversité, prévention des risques naturels, préservation des ressources vitales pour l'homme).

15 Cette pluralité d'usages et d'objectifs nouveaux dans les espaces ruraux devient la source d'enjeux et de tensions entre différents groupes d'usagers (PERRIER-CORNET, 2002b).

1.2. Le cadre de vie : un bien collectif mixte sous l'influence des pouvoirs publicsLe cadre de vie

16 Le cadre de vie n'est pas un bien économique standard respectant les conditions de substituabilité, d'homogénéité et il n'existe pas de marché pour un bien si complexe à l'instar du patrimoine (GREFFE, 1990). Les individus et les collectivités affectent des ressources à la recherche d'un cadre de vie de qualité et procèdent par conséquent à des choix alternatifs. La combinaison des services rendus par les biens supports conduit à différents outputs dont le cadre de vie résidentiel.

Les biens environnementaux localisés, constitutifs du cadre de vie ne sont pas dédiés à un usage exclusif. Ils ont une localisation particulière, ils sont complexes, multifonctionnels et marqués par différents statuts juridiques et économiques (ALLAIRE et BLANC, 2001). Ce sont pour la plupart des actifs et des ressources spécifiques (COLLETIS et PECQUEUR, 1995). À l'instar des travaux de TORRES (1998) sur le cadre de vie urbain, le cadre de vie rural et périurbain est composé d'une infrastructure matérielle et informationnelle. L'infrastructure matérielle combine différents objets attachés à l'espace: des biens économiques privés (ils sont produits, reproductibles, substituables). Ils entrent dans des transactions marchandes pour la plupart ; des biens collectifs généralement fournis par la puissance publique locale ; des actifs naturels. Il s'agit de biens ni produits, ni reproductibles, assimilables à des d'actifs naturels multifonctions n'ayant pas de substitut étroit en ce qui concerne leur consommation directe et ne pouvant être reproduits par des moyens techniques. L'infrastructure informationnelle est l'ensemble des règles qui s'appliquent sur les biens supports et régissent leurs usages. Ces règles sont présentes par exemple dans les différents codes: code rural, code de l'environnement, code de l'urbanisme, dans la pure tradition du droit français écrit. Mais il s'agit également de règles d'usage moins formelles ou de routines.

De plus, les biens constitutifs du cadre de vie sont dotés d'une multitude d'attributs (LANCASTER, 1966), et chacune des dimensions d'un bien environnemental localisé ne pourra correspondre parfaitement à un droit d'usage qui respecterait stricto sensu les principes d'exclusivité et de transférabilité. Le bien environnemental localisé sera ainsi doté d'une multitude de droits. La notion de droit d'usage parfois malencontreusement 
appelée droit de propriété renvoie à la notion de property right qui se rapporte au droit de propriété fonctionnel du bien. Il s'agit de droits subjectifs, socialement reconnus et qui ne se rapportent pas à l'appropriation privée du bien (ownership right) comme KIRAT (p. 63, 1999) le précise : "nous pouvons avoir un droit à respirer un air pur (un property right), mais nous ne sommes pas propriétaire de l'air que nous respirons". Alors en présence de biens dont les droits de propriété sont incomplets, et devant la difficulté à rendre un droit d'usage exclusif - donc de le transférer comme les conditions de l'échange marchand l'exigent la rencontre d'activités contradictoires risque de déboucher sur le conflit.

La fourniture politico-administrative des biens et des droits d'usage constitutifs du cadre de vie

19 Si certains biens sont co-produits par l'investissement, les services environnementaux, ou la production des ménages, rares sont les situations de développement local dont la concrétisation échappe à l'intervention de la puissance publique, qu'il s'agisse des collectivités territoriales, des services déconcentrés de l'Etat ou de l'administration centrale. L'intervention publique est essentielle pour répondre à la demande de fonctionnalités rendues par les biens environnementaux localisés. L'action des pouvoirs publics dont l'objectif n'est pas forcément de produire intentionnellement un cadre de vie se décline selon deux grandes modalités : la fourniture d'infrastructures publiques et la fourniture de règles d'usage.

20 Les collectivités locales et l'Etat exercent un pouvoir réglementaire important qui s'applique à l'ensemble des biens support de l'infrastructure matérielle du cadre de vie. Conception fondée sur la tradition régalienne de l'Etat, elle soutient que les politiques publiques en matière d'urbanisme, d'environnement ou d'agriculture doivent reposer sur des règles contraignantes de type administratif. Ce sont des instruments qui limitent quantitativement les actions des agents économiques. Ce mode de régulation par les quantités passe par l'application de règles d'usage soumises à des objectifs, des procédures et des outils qui s'appuient très fortement : sur le régime des Installations Classées pour la Protection de l'Environnement (ICPE) ; sur les zonages (POS désormais Plan Local d'Urbanisme, Plan de Prévention des Risques Naturels (PPRN), NATURA 2000...) ; sur les permis de construire et sur les procédures d'expropriation ; sur les schémas directeurs (assainissement, carrière, déchets...) ; sur la conduite d'enquête publique, la déclaration d'utilité publique lors de la création ou l'extension d'ouvrages ou d'infrastructures diverses au nom de l'intérêt général.

21 Par leurs actions (production, consommation) sur les biens environnementaux localisés, l'ensemble des agents changent l'état de qualité globale du cadre de vie. Et parmi eux, deux types d'agent que nous qualifions d'autorité publiques locales interviennent plus particulièrement dans la fourniture des règles d'usage précédemment citées : d'une part, les collectivités locales, pour l'essentiel les communes, sont compétentes dans le domaine des règles d'urbanisme et, à ce titre, interviennent soit dans la promotion de dynamiques de développement économique, soit dans celle du cadre de vie sur le territoire de la commune. Issues du vote, les collectivités locales sont les délégataires des préférences manifestées par les électeurs concernant la destinée du territoire communal; d'autre part, les services de l'Etat, qu'il s'agisse des services déconcentrés, ou du préfet, agissent en considération de l'intérêt général et sont, à ce titre, les opérateurs de la gestion des intérêts privés face à l'intérêt public, et sont de plus avec l'administration préfectorale les garants de la légalité des actes des collectivités territoriales et des établissements publics. 
1.3 L'analyse économique des conflits d'usageQuelques éléments de définition du conflit d'usage.

Dans notre recherche, les conflits d'usage dans les espaces périurbains et ruraux combinent une question physique lors de la réception d'un effet dommageable réel ou potentiel à propos de l'usage d'un bien environnemental localisé ${ }^{1}$ et une question institutionnelle lors de la controverse sur la fourniture par les pouvoirs publics locaux de biens ou de règles pour certains usages des ressources localisées. En effet, la montée de l'usage résidentiel semble remettre en cause les usages préférés ou sacrifiés par les autorités publiques locales. Celles-ci paraissent ainsi largement impliquées dans les conflits, en témoignent les nombreuses situations polémogènes opposants les élus locaux ou le préfet en charge de la vie de la cité 2 à des riverains d'une future déviation routière, d'un incinérateur, ou d'une porcherie autorisée à s'agrandir. Dans les années 1970, les oppositions aux projets socialement bénéfiques dans le mouvement général de modernisation du pays étaient le plus souvent assimilées à des manifestations de résistances de forces conservatrices (GERMES, 1979, p 5). Les promoteurs ont actualisé leur discours en créditant de façon péjorative les opposants de nimbistes ${ }^{3}$. Mais les conflits environnementaux contemporains pourraient traduire également une demande de participation accrue du public aux décisions qui concernent leur environnement (autrement que par le vote) (GERMES, 1979; De CHAMPRIS, 1997). Toutefois, nous n'oublions pas que la puissance publique est aussi convoquée comme tiers, pour pacifier la société civile dans sa fonction ancestrale d'arbitrage et plus récemment de médiation.

Enfin, nous retenons une définition générique de la notion de conflit d'usage pour une cadre de vie qui peuvent être selon les situations :

Des conflits environnementaux (CHARLIER, 1999). Il s'agit des conflits déclenchés par les impacts négatifs avérés d'un équipement ou d'une activité à l'origine d'une pollution (cas de l'exploitation de carrières);

Des conflits d'aménagement (DZIEDZICKI, 2001). Ils sont déclenchés par les projets d'aménagement et leurs impacts négatifs potentiels. Ils ne contiennent pas de menaces de pollutions mais seulement des nuisances visuelles (cas des projets éoliens);

Des conflits d'implantation (DZIEDZICKI, 2001). Ils sont déclenchés par un projet contenant des activités à l'origine de nuisances ou de pollutions comportant des risques de toutes sortes (cas de l'implantation d'incinérateurs);

Les conflits d'usage dans leur acception économique étroite. Ils sont déclenchés dans le cadre d'usages concurrents pour une même ressource (surexploitation d'un hydrosystème) (POINT, 1997);

Enfin les conflits d'accès (MICHEL, 2003). Ils sont déclenchés par l'accès à des espaces dont le statut juridique limite en principe l'usage (cas des conflits entre des randonneurs et propriétaires fonciers).

L'origine physique du conflit d'usage : la présence de biens collectifs joints

De manière générale, les conflits d'usage des actifs ou des ressources localisées signalent la présence d'effets externes technologiques négatifs induits par des positions de proximité géographique dans l'espace entre les agents concernés (CARON et TORRE, 2002).

30 Dans une économie de marché régie par les règles de l'Etat de droit, le marché est perçu comme l'état naturel d'organisation de la société. Le marché est un mécanisme moral permettant de réaliser sans heurt l'interaction de $n$ entités autonomes qui poursuivent 
des logiques différentes (CARRIER, 1993). Or, certains biens ou services collectifs ne respectent pas les conditions d'échanges optimales, provoquant ainsi des défaillances du marché comme les effets externes caractérisés par PICARD (1998, p. 506) : "des situations où les décisions de consommation ou de production d'un agent affectent directement la satisfaction ou le profit d'autres agents sans que le marché évalue et fasse payer ou rétribuer l'agent de cette interaction."

31 À l'origine de l'effet externe existe une offre ou une consommation conjointe d'un bien collectif $g$ résultant des conditions technologiques de production ou de consommation d'un bien privatif $k p$ ou collectif $k c$ régi, lui, par les règles de décision du système d'échange (CATIN, 1985 ; MISHAN, 1969 ; MUSGRAVE, 1968). L'effet est transmis sans prix, sans compensation par un émetteur non intentionnellement. Le produit joint $g$ n'est ni un co-produit, ni un sous-produit faisant l'objet d'une valorisation commerciale. Il s'agit plutôt d'un produit induit non commercialisé (THIEBAUT, 1992). Sans récepteur, point d'externalité technologique, même si un flux physique existe : l'effet externe est une relation entre des agents (THIEBAUT, 1992 ; MOLLARD, 2003). Par ailleurs, dans certaines situations de dépréciations foncières comme c'est le cas pour les zones résidentielles proches d'un aéroport, la présence d'externalités dite pécuniaires pourrait expliquer la situation d'opposition. À notre avis, nous sommes en présence d'externalités dites dynamiques. À l'origine, les riverains reçoivent un effet externe technologique qui dégrade leur cadre de vie : du bruit en plus émis par l'augmentation d'un aéroport par exemple. Dans un second temps, la dégradation du fond sonore qui prévalait pourra avoir un effet dépressif sur le marché immobilier : la présence d'un effet externe pécuniaire est évidente, même si à l'origine l'effet négatif est purement physique ${ }^{4}$. Il dégrade l'intégrité de l'agent et affecte l'utilité du consommateur d'aménités, et le conflit est le mode de signalement de cet effet physique.

Surtout, l'effet externe révèle le défaut d'information concernant les préférences des agents qui ne sont plus en mesure d'orienter leurs décisions de production et de consommation par l'absence d'un signal prix (CATIN, 1985). En principe, le régulateur intervient par des signaux fiscaux (taxes, subventions) pour modifier le comportement de l'émetteur, sans que soient exclus d'autres modes de signalement de la réception de l'effet. En effet, le récepteur pourra être tenté d'infléchir les actions de l'émetteur en lui signalant explicitement par la prise de parole la réception de l'effet externe négatif. Le conflit est pour nous cette situation de signalement de la réception d'un effet externe technologique négatif. C'est le cas de nombreux conflits du cadre de vie où les usages autorisés par les services collectifs rendus par les biens collectifs mixtes en jeu manifestent des effets externes. Si nous n'étions qu'en présence de biens privatifs purs, le marché allouerait efficacement les ressources selon la théorie des droits de propriété. Et, si nous n'étions qu'en présence de biens collectifs purs, aucun heurt ne serait possible.

L'origine institutionnelle du conflit : les problèmes de fournitures des biens et des droits d'usage

Parmi les diverses possibilités d'organisations sociales en France, l'intervention publique est privilégiée pour réguler les usages des biens environnementaux localisés. La puissance publique peut éprouver des difficultés à organiser l'utilisation optimale d'un bien collectif mixte dans un système d'échange faute de droit de propriété défini. En effet, les élus et leurs administrations chargés d'organiser la vie de la cité sont amenés à prendre des décisions qui ne satisferont pas forcément tous les citoyens, même si elles sont fondées sur la recherche de l'intérêt général. Pourquoi? 

agents. Or, si les coûts des effets des décisions des autres sont nuls, d'autres coûts dits de négociation inhérents à la recherche de l'unanimité existent. Ces coûts seront d'autant plus élevés que le nombre de participants à l'accord sur le choix politique sera important. Ainsi, en présence de grands groupes, la délégation de pouvoir s'impose, et si elle permet de réduire les coûts de négociation, elle entraîne des coûts externes liés aux risques de spoliation d'une partie des agents dont les préférences ne seront pas satisfaites lors de l'action publique. Les mandataires vont ainsi transmettre un effet externe négatif de leur décision aux mandants : les citoyens reçoivent ainsi des effets externes politiques négatifs (CATIN, 1985 ; JARRET et MAHIEU, 1998 ; SCHMID, 1978), assimilables aux coûts externes de la décision collective observés par BUCHANAN et TULLOCK (1962), lorsque les électeurs ne peuvent exercer un contrôle direct sur les décisions de leurs représentants qui, mal informés, agissent contre leurs intérêts. La contestation qui s'enclenche, en jouant le rôle de vecteur informationnel, initie un processus de marchandage visant à ajuster la fourniture des droits d'usages des biens collectifs mixtes du rural. L'expression des préférences des citoyens résidents ne peut se satisfaire d'un seul vecteur correspondant à la prochaine échéance électorale (VIGOUROUX, 1999) et ne peut que difficilement se réaliser dans une stratégie de déménagement (exit) (TIEBOUT, 1956), eu égard à l'attachement au lieu de vie (propriété résidentielle, proximité au centre d'emploi, intérêt pour les aménités). Par conséquent, le fonctionnement du contrôle indirect du régime représentatif (vote) est complété par le contrôle direct des représentants (voice) (HIRSCHMAN, 1970). La prise de parole par des groupes de pression, ou devant un tribunal s'inscrit dans un processus d'équilibration censé tendre vers un état de satisfaction collective meilleur. En définitive, le conflit, s'il signale la réception d'un effet dommageable technologique par un agent, se pose également en termes d'ajustement du processus de reconnaissance de fonctionnalités d'une ressource en jeu dans un cadre institutionnel de type juridico-politique.

2. Cadre analytique et méthodologie de traitement des conflits d'usage

\section{Nous définissons le conflit d'usage pour un cadre de vie comme une situation d'opposition déclarée} entre deux catégories d'agents (individus ou groupes d'individus) dont les intérêts divergent à un moment précis lors de l'usage de biens localisés à dimension environnementale et territoriale (sols, espaces naturels, ressources naturelles, aménités) dans les espaces ruraux et périurbains, constitutifs du cadre de vie. C'est la dimension physique. De plus, cette opposition met en scène des agents usagers du cadre de vie et la puissance publique locale contestée pour son action de fourniture de biens publics ou de règles d'usage. C'est la dimension institutionnelle.

retenons le concept d'effet externe technologique, ce n'est pas tant en termes de défaillance de marché, qu'en termes de caractéristiques de l'origine physique. Il s'agit d'identifier la relation d'échange, la nature économique du bien en jeu, la nature du concernement collectif, l'hétérogénéité des parties concernées, les problèmes liés à l'information... Par ailleurs, la notion d'externalité politique permet de saisir le processus de négociation sur les droits de propriété influencé par l'intensité de la négociation politique, et de comprendre les questions de redistribution des droits de propriété.

2.1. Première grille : l'effet externe technologique

La grille des externalités technologiques caractérise la relation entre récepteur et émetteur sur le plan physique (CATIN, 1985). Les variables clés de notre grille d'analyse sont: la notion de concernement collectif hétérogène ou homogène; le statut du bien joint à l'origine de l'effet sur le récepteur en termes de rivalité et d'exclusion. 

cas-types de conflits caractérisés par la nature de l'externalité technologique : Externalité d'accès : il s'agit des situations où l'exclusion est possible par une barrière à l'entrée dans un groupe d'usager. C'est le cas des conflits entre des randonneurs et les propriétaires d'un site remarquable; Externalité d'encombrement ou de surexploitation ${ }^{5}$ : il s'agit de conflits entre une même catégorie d'usagers qui se gênent mutuellement lors de leurs usages. Il n'y a pas d'exclusion possible, mais usage d'une ressource divisible. C'est le cas de conflits entre des associations cynégétiques sur un même territoire de chasse; Externalité de pollution ou de voisinage : il s'agit de situations où le concernement est hétérogène. Celui qui transmet la nuisance n'a pas sa fonction-objectif affectée. C'est le cas emblématique des pollutions comme lors de l'épandage d'effluents d'élevage à l'origine de mauvaises odeurs pour les riverains.

2.2. Deuxième grille d'analyse : l'effet externe négatif de la décision publique types de conflits caractérisés par la nature de l'externalité politique. émetteur sur le plan politique. Les variables clés de notre grille d'analyse sont : l'existence ou non d'un émetteur de l'externalité politique ; le statut de l'émetteur et du récepteur de l'externalité politique ; l'action de l'émetteur de l'effet externe politique : maîtrise d'œuvre, d'ouvrage, mise en application de règles ; les logiques d'action (pression médiatique, recours devant les juridictions administratives ou judiciaires). justice pour trancher le litige. C'est le conflit entre particuliers, typique des troubles de voisinage (résident vs résident ou résidents vs firme). Nous sommes uniquement en présence d'un effet externe technologique.

2.3 La méthodologie utilisée pour tester nos grilles d'analyse des conflits d'usage

Nous avons choisi le département du Puy de Dôme comme terrain d'étude. Le choix de l'échelle départementale a été dicté par la proximité du terrain au site de travail (ENITA Clermont) et parce qu'il est une entité cohérente et un échelon territorial de référence de nombreuses institutions.

Le choix du département du Puy de Dôme est surtout justifié par les caractéristiques de sa dynamique économique et démographique. Le Puy de Dôme est un département à l'image de nombreux autres avec un étalement urbain révélateur d'une montée de l'économie résidentielle. Les tendances lourdes de métropolisation et de périurbanisation engagées depuis 40 ans se poursuivent, mais à un rythme beaucoup plus faible autour de la 
métropole régionale Clermont-Ferrand ( $43 \%$ des habitants du département vivent dans l'agglomération clermontoise) et, plus précisément, le long d'un axe d'accès à l'emploi très peuplé orienté nord-sud adjacents aux autoroutes A71 et A75. L'emploi est concentré sur cet axe grâce à la présence d'industries multiples et nombreuses, et du tertiaire. Par conséquent, la population du Puy-de-Dôme travaille et habite essentiellement sur cet axe, ce qui entraîne une forte pression humaine pour l'usage des ressources du territoire. De plus, l'agriculture est encore très présente et puissante sur toute la campagne. Par ailleurs, avec plus de la moitié des nuitées marchandes (6 millions en 2003) de la région Auvergne (SPOT, 2003), le Puy de Dôme confirme sa forte fréquentation touristique. Les paysages originaux et variés ainsi que les sites naturels et patrimoniaux remarquables font du département un lieu de villégiature couru, ce qui ne fait qu'exacerber les concurrences entre usagers.

Deux sources ont été mobilisées afin de rassembler un matériau empirique exploitable sur les conflits concernant uniquement les espaces ruraux et périurbains au sens du Zonage en Aires Urbaines et en aires d'Emploi de l'espace Rural (ZAUER) de l'INSEE.

Nous avons récolté dans un premier temps des articles de presse dans le quotidien régional La Montagne. La recension a été effectuée pour une période de plus de trois ans (janvier 1999 à février 2002), en consultant près de 1600 éditions du Puy de Dôme.

Nous avons choisi d'exploiter dans un second temps cinq années (janvier 1998 à décembre 2002) de contentieux du Tribunal Administratif (TA) de Clermont-Ferrand en consultant plus de 1000 jugements. Il ne s'agit pas de discuter le comportement du juge ou le contenu des jugements en termes d'interprétation du droit législatif ou réglementaire. L'analyse d'une partie de l'activité d'un TA et de la Presse Quotidienne Régionale (PQR) est une voie capable de dater, de localiser, et d'exposer les caractéristiques d'émission et de réception des effets externes à l'origine des conflits.

51 Le recueil des jugements et des articles de presse n'a porté que sur les conflits mettant en jeu l'usage de ressources localisées des espaces ruraux et périurbains. Les conflits d'usage du TA ont été recensés à partir de six postes de la nomenclature : agriculture, environnement, expropriation, domaine-voirie, police, et urbanisme.

3. Les premiers résultats3.1 Les conflits d'usage dans la Presse Quotidienne Régionale $(\mathrm{PQR})$ : la défense du cadre de vie par l'action collective

Les 112 conflits recensés dans la $\mathrm{PQR}$ touchent de nombreux thèmes (cf. Tableau 1): Transport, chasse et pêche, déchets, carrière, agriculture, eau, énergie, biotope, risques naturels et industriels, urbanisme, loisirs, droit de propriété, habitat. 
Tableau 1. Grands thèmes de conflits recensés dans la presse quotidienne régionale

\begin{tabular}{|l|r|r|}
\hline \multicolumn{1}{|c|}{ Thématiques des conflits dans la Montagne 1999/2002 } & Nb. cit. & Fréq. \\
\hline implantation activités "à risques" (déchets, explosif́s...) & 32 & $28,6 \%$ \\
\hline implantation ou fonctionnement d'une infrastructure de transport & 18 & $16,1 \%$ \\
\hline implantation, extension de carières & 15 & $13,4 \%$ \\
utilisation de foncier & 15 & $13,4 \%$ \\
pollution diverses d'origine industrielle ou agricole & 12 & $10,7 \%$ \\
mode de gestion des animaux péchés et chassés & 9 & $8,0 \%$ \\
restriction d'accès à un bien collectif & 7 & $6,3 \%$ \\
\hline autres & 4 & $3,6 \%$ \\
\hline TOTAL OBS. & 112 & $100 \%$ \\
\hline
\end{tabular}

53 L'analyse du matériau empirique nous indique que les usagers du cadre de vie (récréatifs et résidentiels) sont les plus concernés comme récepteurs d'effets externes négatifs technologiques (71\% des cas) et s'affrontent aux usagers productifs ou de nature (usage de réservation), voire entre usagers du cadre de vie. Un cas sur cinq concerne des usagers productifs contestant d'autres usagers. Enfin, les protecteurs de la nature ne sont concernés que par 12 situations dans lesquelles ils mettent en cause d'autres usagers au nom de la conservation des milieux et des espèces. Nous constatons par ailleurs que les activités productives (agriculture, extraction de ressources naturelles, stockage de déchet, production d'énergie, industrie) sont les plus mises en cause (70\% des cas). La forme la plus courante de conflit est caractérisée par une opposition entre des usagers du cadre de vie et des usagers productifs (un cas sur deux).

Il ressort de l'application de la grille d'analyse des effets externes technologiques que les usagers poursuivent des buts antagonistes et rarement le même but de manière compétitive, signes de la multiplication des usages pour des ressources multifonctionnelles. En effet, dans plus de $80 \%$ des conflits, le concernement collectif est hétérogène. À l'origine de l'effet externe, un émetteur en consommant ou en produisant un bien, produit ou consomme conjointement un bien collectif environnemental localisé et affecte les autres usagers de cette ressource sans jamais être atteint par l'impact de ses usages sur sa fonction-objectif principale. Dans la majorité des cas, 82 situations sur 112, il s'agit de ce type d'externalité dite de pollution.

Les autres situations sont moins nombreuses (15\%) et concernent les conflits avec concernement collectif homogène, typiques des situations de concurrences pour une ressource naturelle entre usagers ayant la même fonction pour un même but.

3.2 Le contentieux administratif : des conflits entre des particuliers et les pouvoirs publics pour la défense des attributs du cadre de vie

Si la $\mathrm{PQR}$ relate de nombreux conflits entre des agents organisés collectivement défendant les attributs de leur cadre de vie contre les décisions des responsables élus ou mandatés, l'analyse du contentieux administratif présente une image différente du contentieux. Le TA est un espace de régulation des conflits à travers lequel sont contestées les décisions des autorités publiques locales (le préfet et les collectivités locales) mais à la différence de la $\mathrm{PQR}$, les requérants sont essentiellement des particuliers et des entreprises ( $72 \%$ des requérants). Nous constatons qu'un peu moins d'un cinquième des requêtes est porté par des organisations collectives. 
57 Sur 5 ans, 294 conflits d'usage ont été recensés à partir de 380 jugements, ce qui correspond à près de $8 \%$ des affaires du Puy de Dôme traitées par le TA. Les jugements relatent, à l'instar de la $\mathrm{PQR}$, une grande variété de situations contentieuses concernant l'urbanisme, les remembrements, les infrastructures de transport, les installations classées agricoles, le traitement des déchets, les carrières, les activités touristiques, les espaces naturels. La défense du cadre de vie est la fonction faisant l'objet du plus grand nombre de requêtes (58\%).

58 Le contentieux administratif donne une information précise sur l'objet de la requête. Il s'agit dans la majorité des cas de la contestation d'une décision prise par les pouvoirs publics. Seules une vingtaine de situations concernent la requête des autorités publiques contre des usagers bafouant l'intérêt général. Surtout, derrière ces décisions (arrêté municipal, arrêté préfectoral), nous identifions des dispositifs réglementaires dont l'objet est d'organiser les droits d'usages : des dispositifs d'organisation générale des usages par le zonage (PLU, PPRN) ; des dispositifs de modification des activités à dimension spatiale (remembrement, permis de construire, servitudes diverses, code de la route locale...) ; l'octroi de droit à polluer ou à ne pas être pollué avec l'application de la réglementation sur les ICPE ; l'action de fourniture de biens publics (tracé de routes, gestion des déchets, assainissement...).

3.3. Attentes des contestataires et impact du conflit d'usage : le contestataire cherche l'abandon de l'usage indésirable

Qu'il s'agisse des conflits repérés dans La Montagne ou au TA, les requérants escomptent majoritairement l'abandon ou l'aménagement de l'usage contesté (93\% des requêtes au TA et $95 \%$ des attentes observées dans la presse). Les contestataires n'attendent pas de compensation financière alors qu'elle est un des principes fondamentaux des marchandages en économie.

Dans la $P Q R$, les contestataires attendent d'un maire, du préfet ou d'une autre autorité qu'une injonction à cesser un usage soit exprimée contre le mis en cause, ce qu'ils obtiennent le plus souvent. En effet, dans $65 \%$ des cas, les contestataires obtiennent une solution satisfaisante : l'activité indésirable est abandonnée, délocalisée ou aménagée. L'impact spatial de la contestation est une réalité, mais il nous est difficile de conclure à un effet de spécialisation résidentielle de certains espaces périurbains et ruraux.

$61 \mathrm{Au} \mathrm{TA}$, les requérants attendent également qu'une injonction à cesser l'activité jugée indésirable soit émise par le juge administratif à l'encontre des défendeurs. Le juge suit parfois les requérants. Ceux-ci obtiennent dans le cadre de la prévention de projet, l'annulation d'un permis de construire ou une autorisation d'exploiter, mais plus rarement le juge ordonne la cessation des nuisances d'une installation classée en activité.

Les metteurs en cause obtiennent plus facilement gain de cause lorsqu'ils sont regroupés en association ou s'ils sont épaulés par un avocat. La sous-dotation en connaissance des moyens de droit est caractéristique des particuliers isolés dont les requêtes sont majoritairement rejetées. L'issue d'une requête est deux fois sur trois négative pour le requérant. Pour autant, le conflit ne s'arrête pas là. Le recours en contentieux administratif n'est pas la solution ultime, il n'est qu'un épisode du processus conflictuel, et la décision rendue par le juge peut dans certains cas ne trouver aucune application sur le terrain.

3.4. Le conflit d'usage : mécanisme de révélation de préférences et de marchandage politique pour la redistribution des droits d'usages 
L'analyse dans la PQR des différentes formes de conflits d'usage montre que les autorités publiques locales sont très présentes dans les conflits selon 4 grandes catégories que nous avons construites à partir de notre grille des effets externes politiques et confirmée par une Classification Ascendante Hiérarchique :

4 soit parce qu'elles sont directement mises en cause quand elles fournissent directement un bien public ; soit parce qu'elles sont indirectement mises en cause, car en prenant des décisions elles transmettent des effets externes politiques négatifs;

soit parce qu'elles sont invitées à user de leur pouvoir de contrôle et/ou de police à l'encontre d'un usager dont les activités sont jugées indésirables ;

6 soit enfin parce qu'elles sont sollicitées pour accompagner le processus de redéfinition des droits d'usage.

Parmi les 112 situations conflictuelles repérées dans la PQR, nous n'entrevoyons que quelques rares situations où la puissance publique est absente.

8 Première catégorie : L'autorité publique locale : émettrice d'effets externes technologique et politique négatifs simultanés (schéma 1 ).

Des conflits surviennent quand une autorité publique locale (un conseil général via le préfet ou une commune) exerce sa maîtrise d'œuvre ou d'ouvrage (fourniture d'infrastructure) comme nous l'avons observé dans 27 cas.

Schéma 1

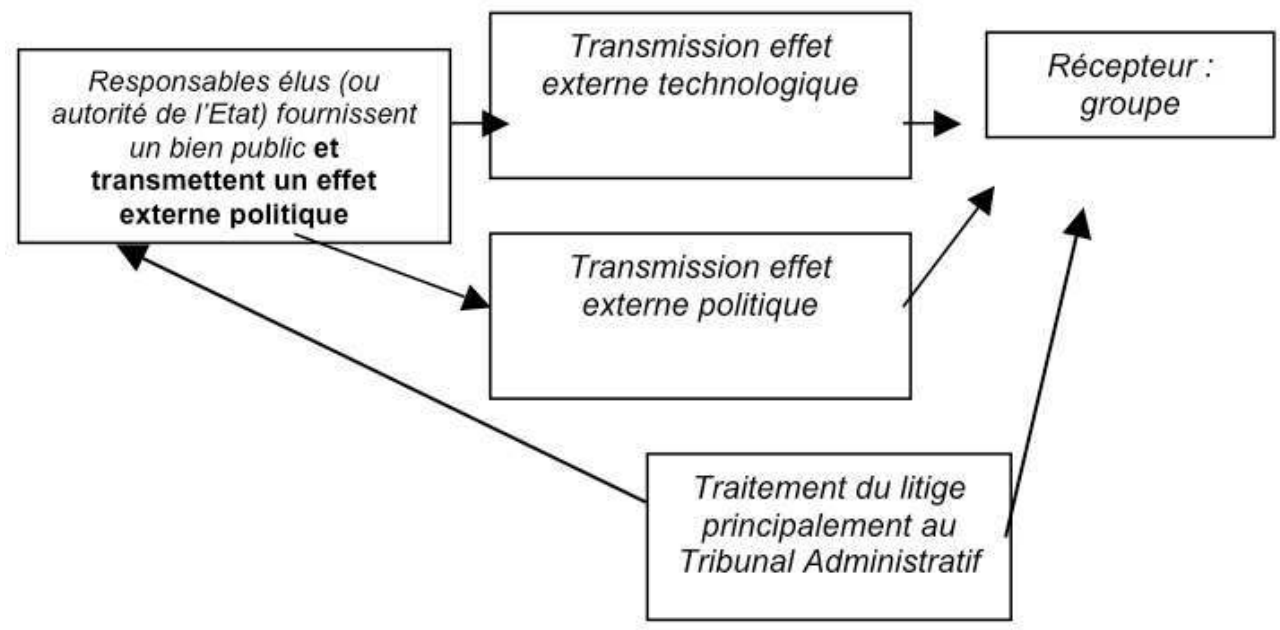

70 Le conflit d'implantation d'un incinérateur dans le Puy de Dôme représente le cas emblématique de cette première catégorie. De nombreuses associations contestent l'action d'un syndicat mixte départemental chargé d'organiser pour le compte de plus de 500 communes le traitement des déchets. Le syndicat est le maître d'ouvrage des unités de valorisation énergétique et biologique. Il transmet pour les opposants un effet dommageable physique potentiel à l'origine du conflit. Le danger pour la santé humaine est l'argument principal invoqué par les riverains. Dans le même temps, parce qu'il ne connaît qu'imparfaitement les préférences des riverains, le syndicat par ses choix non conformes aux attentes des riverains transmet un effet externe politique négatif. Les contestataires rejettent la localisation du projet et ses dommages supposés par tous les moyens (pétitions, manifestations de rue, débats publics, site internet dédié). En outre, les opposants ont tenté d'activer le levier juridique en émettant des avis défavorables à la 
Déclaration d'Utilité Publique (DUP) visant à modifier le PLU nécessaire à la localisation de l'incinérateur. Ce levier d'action aurait pu permettre aux opposants d'obtenir l'abandon du projet ou la délocalisation de l'infrastructure eu égard aux conclusions défavorables du commissaire enquêteur pour la DUP, mais le Conseil d'Etat n'a pas suivi cet avis, en autorisant le syndicat mixte à poursuivre le processus : le conflit continu...

Deuxième catégorie : L'autorité publique locale : émettrice d'un effet externe politique négatif (schéma 2)

La responsabilité d'une autorité publique (le préfet ou un maire) est engagée dans le cadre de l'application des autorisations d'exploiter octroyées aux entreprises (cas des ICPE, attribution de permis de construire) comme nous l'avons observé dans 29 cas. Dans ces derniers, une firme ou un particulier transmet un effet externe technologique parce qu'une autorité publique autorise son activité jugée indésirable par le récepteur. La puissance publique locale transmet pour sa part un effet externe politique négatif comme dans la première catégorie quand elle applique des dispositifs qui permettent d'encadrer les droits à polluer (cas des ICPE) (cf. schéma 2).

\section{Schéma 2}

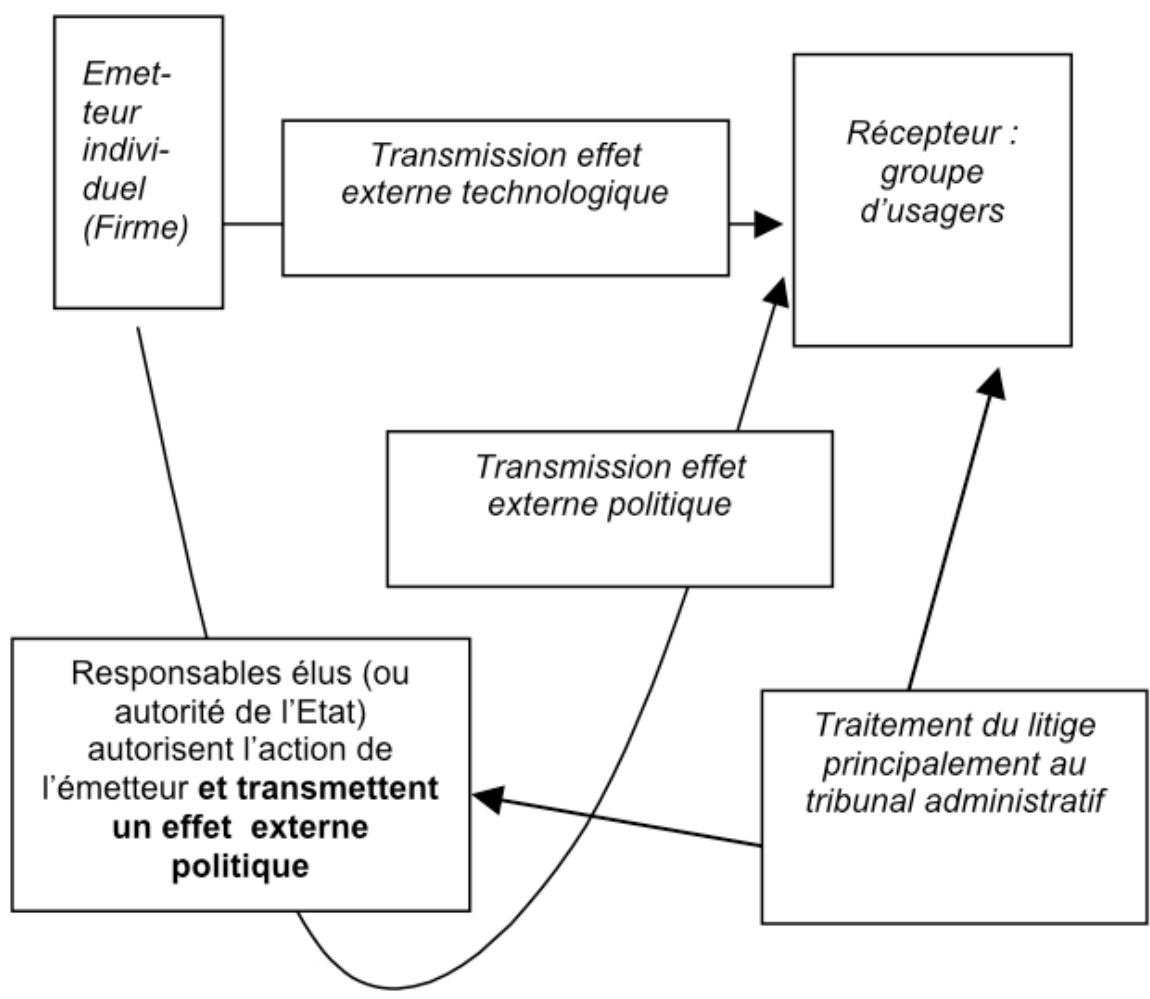

Les de modifier les pratiques de l'émetteur :

Ils requièrent au TA et obtiennent, lorsqu'elle n'était pas respectée, l'application de la réglementation. Cela conduit par exemple une entreprise de stockage de déchets à réduire l'activité du site afin de respecter les règles de fonctionnement d'un Centre de Stockage de Déchets Ultimes de classe II.

Dans d'autres situations, la requête des opposants est infructueuse devant le TA car les motifs et/ou les moyens de droit invoqués ne sont pas suffisants. Les pouvoirs publics locaux peuvent se trouver mis hors-jeu par des opposants déterminés, qui par la pression sur l'émetteur de l'effet externe technologique obtiennent l'abandon ou la modification 
d'une activité indésirable. C'est le cas par exemple d'un porcher alors qu'ayant obtenu le droit d'exploiter une installation classée, se voit contraint sous la pression des riverains d'abandonner son projet d'extension autorisé par le préfet...

Dans d'autres situations encore, parce que les coûts d'enforcement de la réglementation (coût de contrôle, de sanction) sont finalement trop élevés pour la puissance publique locale, un marchandage entre émetteur et récepteur de l'effet externe technologique négatif est mis en œuvre et conduit à produire de nouvelles règles d'usage ou de nouvelles pratiques. C'est le cas d'un conflit entre les habitants d'un village qui contestent l'autorisation d'exploiter une carrière validée par les services de l'Etat parce que responsable d'une augmentation du trafic routier et des nuisances liées. Le marchandage entre les riverains et le carrier conduit ce dernier à déplacer l'accès au site d'extraction afin d'éviter la traversée du village.

Troisième catégorie : Les autorités publiques locales : contestataires sous la pression des citoyens (schéma 3)

Dans cette catégorie, nous montrons que les autorités publiques contestent l'action d'un particulier ou d'une firme, voire d'une autre autorité publique qui ne respecte pas les droits d'usage (22 situations). Par exemple, le maire, sous la pression de ses administrés, conteste le projet de création d'un centre de stockage d'explosifs en refusant l'attribution d'un permis de construire. Les opposants en utilisant différents moyens de pression, notamment en convoquant la $\mathrm{PQR}$ et en faisant circuler une pétition, ont pu accéder via le maire à l'instrument juridique ad hoc pour empêcher le projet : le permis de construire.

Schéma 3

Schéma 3

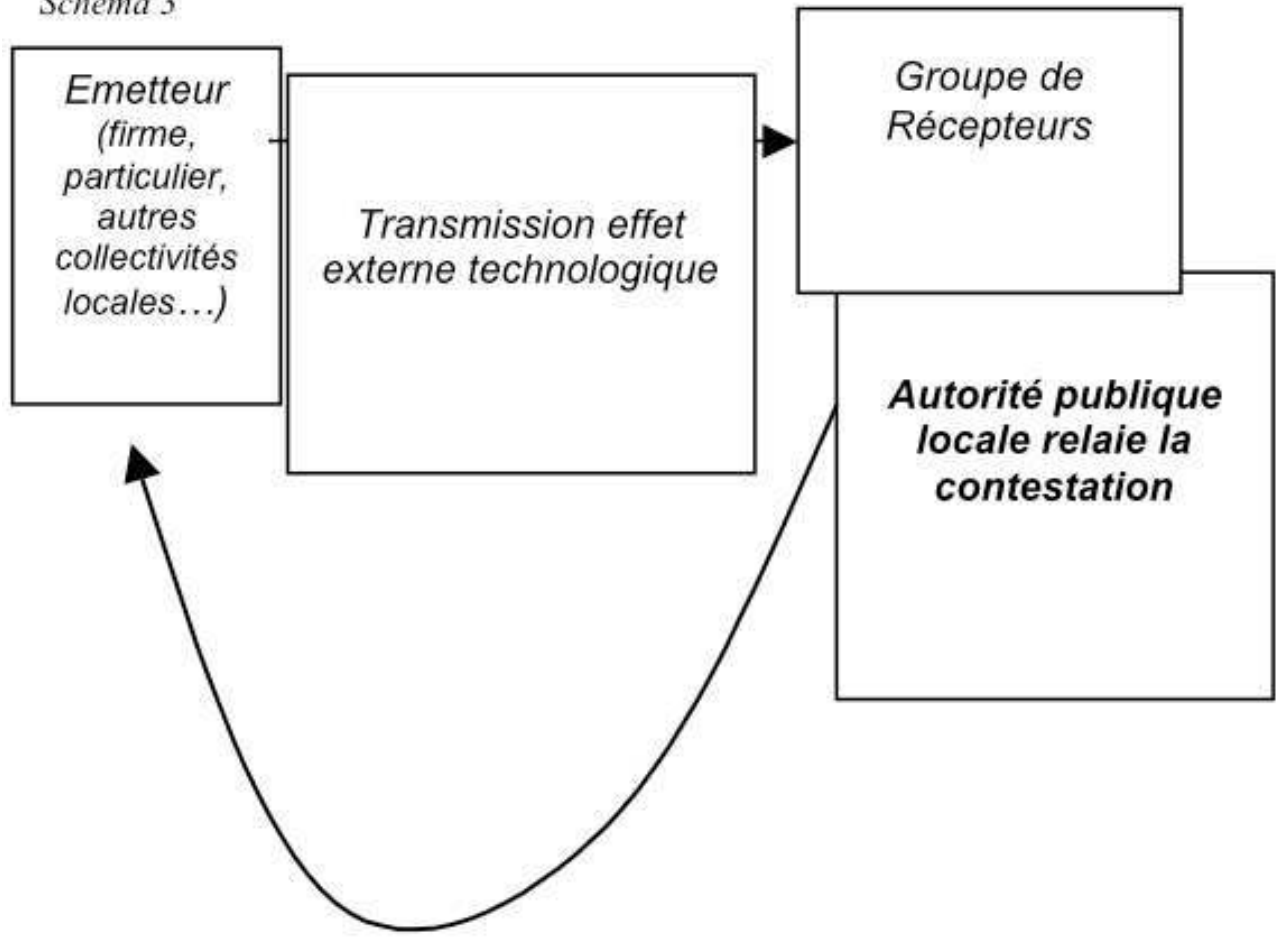

Quatrième catégorie : Les pouvoirs publics locaux accompagnateurs de la définition des droits d'usage (schéma 4) 
80 Cette dernière catégorie concerne des conflits interindividuels pour lesquels apparemment les autorités publiques locales ne sont pas concernées. Dans cet ensemble, nous dénombrons en fait près de 34 situations dites de conflits interindividuels. Elles opposent des particuliers et des firmes. Or dans la moitié des cas, la puissance publique locale est appelée à faire appliquer les termes du "contrat" qui définissent les droits de chaque partie en conflit, ou à proposer une solution qui pourra se traduire par la prise en charge de coûts de contrôle des usages, voire par le financement d'un dispositif technique.

Schéma 4

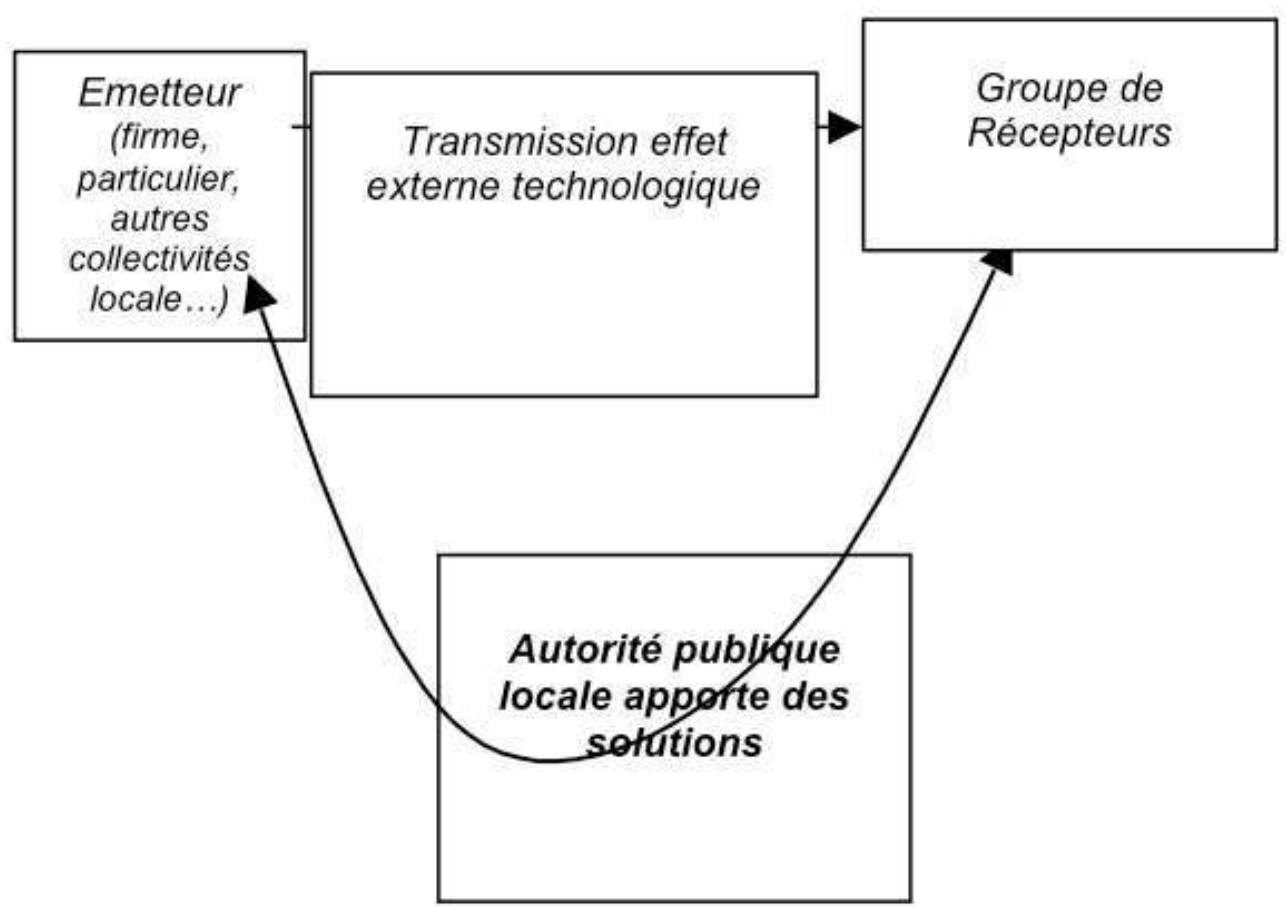

81 Une commune est par exemple invitée à imaginer une nouvelle signalétique permettant d'éviter les nuisances routières consécutives au développement de l'activité d'une entreprise de conditionnement d'eau de source. La portion de route défendue par les riverains - bien commun initialement partagé entre résidents automobilistes et transporteurs routiers - prend le statut de voie domestique et s'affirme comme un bien club. Dans ce cas également, nous observons une stratégie de saisine d'un instrument de droit (le code de la route communale) par les opposants qui permet in fine de faire reconnaître un droit à une voie routière plus sûre et moins bruyante.

4. Quels enseignements tirer ?4.1 Le conflit d'usage pour un cadre de vie : la mise en cause des usages productifs privilégiés

82 Les conflits d'usage pour un cadre de vie représentent la catégorie la plus souvent exprimée devant le TA ou dans la PQR. Les actions plus ou moins démonstratives des défenseurs du cadre de vie leur permettent dans de nombreux cas d'exprimer leurs prétentions. Prétentions que l'on pourrait assimiler dans l'analyse coasienne à la revendication d'un droit d'usage fonctionnel réciproque : un property right. Mais, il existe un décalage entre les prétentions des agents notamment les usagers du cadre de vie, et le droit codifié appliqué par le juge administratif. La montée de l'économie résidentielle exprimée dans la périurbanisation et la reconquête du rural, semble remettre en cause les 
droits d'usages préférés par les agents, et entérinés historiquement dans les règles et les décisions publiques. Nous interprétons les requêtes des usagers du cadre de vie comme des actions destinées à convaincre les autres utilisateurs potentiels des biens environnementaux localisés d'accepter un autre usage, et la règle correspondante. Le droit d'usage est une convention entre individus à propos de choses ou d'autres individus. Plus les usages alternatifs seront possibles et sources de profits, plus le coût à affronter les intérêts concurrents sera élevé, et moins le droit exclusif sera absolu. N'est-ce pas le cas des nombreux conflits entre usages productifs et usages résidentiels qui ne font que révéler les difficultés des producteurs à maintenir leur contrôle ancestral des actifs spécifiques locaux contre la pression des usagers résidentiels visant à faire reconnaître de nouvelles ressources spécifiques?

4.2 Le conflit d'usage pour un cadre de vie : un moyen de saisine des instruments de droits par les résidents

Ce travail met en évidence la faible variété des différents modes d'allocation des ressources territoriales à des usages alternatifs et montre la place centrale des pouvoirs publics locaux dans l'organisation des usages et par conséquent dans les conflits. Plus qu'une remise en cause du rôle de la puissance publique locale, le conflit, par les signaux qu'il émet et par le marchandage politique qui le caractérise, permet d'ajuster et de perfectionner le rôle de la puissance publique en matière d'organisation des règles d'usage sur les biens collectifs localisés. Nous constatons ainsi que le conflit est assimilable à un mécanisme de ré-intégration du citoyen local dans la sphère informationnelle et décisionnelle des politiques publiques locales. La gestion décentralisée des biens collectifs comme l'est un marchandage semble ainsi s'exprimer dans la conflictualité. Les règles d'usage existent et sont très largement élaborées, appliquées et contrôlées par des agents chargés d'organiser la vie de la cité comme le préfet et le maire. Ce sont les deux institutions qui par les arrêtés qu'elles promulguent, transmettent des effets externes technologiques et politiques négatifs. Les responsables élus ou nommés ne sont pas en mesure de connaître suffisamment les préférences des agents ou éprouvent des difficultés à faire respecter des règles d'usages satisfaisant la majorité des usagers. Il n'est donc pas surprenant de voir les usagers chercher à modifier la définition des droits d'usage en contestant et en s'emparant des dispositifs réglementaires dont les responsables usent pour organiser sans heurt la diversité des usages.

4.3 Le conflit d'usage : organisateur spatial des activités?

En définitive, ce travail parce qu'il interroge les dispositifs à l'origine de la redistribution des activités dans l'espace est utile pour comprendre la dynamique de recomposition des espaces ruraux. La défense des intérêts des parties concernées par certaines des dimensions des décisions publiques du développement territorial s'appuie sur la saisine et la contestation des instruments juridiques qui sont à l'évidence les leviers de l'action politique locale. Le processus de marchandage entre les parties et sa médiatisation débouche en majorité sur des solutions non financières comme l'abandon ou la délocalisation de l'activité indésirable. Ainsi à de nombreuses reprises, nous devinons les formes d'une ségrégation spatiale des usages. Les espaces résidentiels semblent de moins en moins tolérer certaines activités productives à l'origine de nuisances et de pollutions. Les conflits joueraient comme des organisateurs spatiaux des activités et pourraient conduire à modifier la physionomie des espaces ruraux et périurbains au nom du cadre de vie. 


\section{BIBLIOGRAPHIE}

ALLAIRE G., BLANC M., 2001, « Local/global institutional systems of environmental public action. » (http://www.toulouse.inra.fr/centre/esr/publi/WP2001.html ) INRA ESR Toulouse Cahier de recherche 2001/21:19p.

Aubert F., Blanc M., 2002, « Activités économiques et emplois : le rural refuge de secteurs déclassés ou milieu attractif ? » in Perrier-Cornet P. (dir.), Repenser les campagnes, Editions de l'Aube, La Tour d'Aigues, 2002, pp. 147-173.

BENARD J., 1985, Economie publique, Paris, Economica, 430 p.

BUCHANAN J., TULLOCK G., 1962, The calculus of consent, Logical foundations of constitutional Democraty, Ann Arbor paperbacks, University of Michigan Press, $361 \mathrm{p}$.

CARON A., TORRE A., 2002, « Les conflits d'usage et de voisinage dans les espaces ruraux : une analyse économique ». in PERRIER-CORNET, P. (dir.), A qui appartient l'espace rural ? Enjeux publics et politiques. La Tour d'Aigues, Editions de l'aube, DATAR: 49-78.

CARRIER B., 1993, L'analyse économique des conflits- éléments d'histoire des doctrines, série économie, Paris, Publication de la Sorbonne, $160 \mathrm{p}$.

CATIN M., 1985, Effets externes -Marché et systèmes de décision collective, Paris, Editions Cujas, 455p.

CAVAILHES J., SCHMITT B., 2002, « Les mobilités résidentielles entre villes et campagnes », in PERRIER-CORNET P. (dir.), Repenser les campagnes, La tour d'Aigues, Editions de l'Aube, pp. 35-66.

CHARLIER B., 1999, « Géographie des conflits environnementaux depuis 1974 » Géographie, Université de Pau: 753.

COLLETIS G., PECQUEUR B., 1995, « Politiques technologiques locales et création de ressources spécifiques » Economie Industrielle et Economie Spatiale. RALLET, A et TORRE, A. Paris, ASRLF: 455-463.

de CHAMPRIS A., 1997, « Les conflits d'aménagement et le syndrome NIMBY, nouvel enjeu du management public », Techniques, territoire et société, n³4, pp. 129-139.

DEVERRE C. et al., 2002, « La question de la nature et ses implications territoriales », in PERRIERCORNET P. (dir.), Repenser les campagnes. La tour d'Aigues, Editions de l'Aube/DATAR. Chapitre 9: 217-238.

DZIEDZICKI J. M., 2001, « Gestion des conflits d'aménagement de l'espace : quelle place pour les processus de médiation? ", Géographie. Tours, Université François Rabelais, Centre d'Etudes Supérieures d'Aménagement: 443.

FAUCHEUX S., NOEL J.-F., 1995, Economie des ressources naturelles et de l'environnement, Paris, Armand Colin, $370 \mathrm{p}$.

GERMES, 1979, « Environnement-conflits-participation », Les cahiers du GERMES(2): 272.

GOFFETTE-NAGOT F., 1996, "Choix résidentiels et diffusion périurbaine", RERU, n² 2, 1996, pp. 229-246.

GREFFE X., 1990, La valeur économique du patrimoine - La demande et l'offre de monuments, Paris, Anthropos-Economica, $253 \mathrm{p}$. 
HIRSCHMAN A.O., 1970, Exit, Voice and Loyalty, Harvard University Press, 144 p.

JARRET M.-F., MAHIEU F.-R., 1998, Economie publique : théories économiques de l'interaction sociale, Paris, Ellipses, 126 p. KIRAT T., 1999, L'économie du droit. Paris, La découverte, 123 p.

LANCASTER K.J., 1966, "A new approach to consumer theory", Journal of Political Economy, nº 74, april, pp. 132-157.

LECAT G., 2004, "En quoi le cadre de vie rural contribue-t-il à expliquer les prix ?" RERU. Revue d'Economie Régionale et Urbaine (1): 29-56.

LE JEANNIC T., 1997, "Trente ans de périurbanisation : extension et dilution des villes." Economie et Statistiques, vol. 7, $n^{\circ}$ 307, pp. 21-42.

MICHEL C., 2003, L'accès du public aux espaces naturels agricoles et forestiers et l'exercie du droit de propriété : des équilibres à gérer. RGTE-ENGREF-CIRED,. Clermont-Ferrand, Ecole Nationale du Génie Rural des Eaux et des Forêts: 511.

MISHAN E.J., 1969, "Property Rights and Amenity Rights", in MISHAN E.J. (dir.), Technology and Growth: The Price We Pay, London, Stables Press, pp. 36-42.

MOLLARD A., 2003, "Multifonctionnalité de l'agriculture et territoires : des concepts aux politiques publiques." Cahier d'Economie et de Sociologie Rurales (66): 27-54.

MUSGRAVE R.A., 1968, "L'offre des biens publics", in Economie publique, Actes du colloque de Biarritz, CNRS, pp. 81-117.

PERRIER-CORNET P., (Dir.), 2002a, Repenser les campagnes, La Tour d'Aigues, Editions de l'aube/ DATAR, $279 \mathrm{p}$.

PERRIER-CORNET P., (Dir.), 2002b, À qui appartient l'espace rural ? - Enjeux publics et politiques, La Tour d'Aigues, Editions de l'Aube-Datar, $141 \mathrm{p}$.

PICARD P., 1998, Eléments de microéconomie - Théorie et applications, 6ème édition, Paris, Montchrestien, $587 \mathrm{p}$.

POINT P., 1997, La valeur de l'eau comme actif naturel multifonction. Compte rendu de l'Académie d'Agriculture de France 83(4): 179-194.

SCHMID A., 1978, Property, power, and public choice: an inquiry into law and economics, New York, Praeger, $316 \mathrm{p}$.

SPOT Auvergne http://www.spot-auvergne.asso.fr , 2003, "Les chiffres clés Auvergne sur l'année touristique 2002". Clermont-Ferrand, SPOT auvergne. accès : janvier 2005.

TIEBOUT C., 1956, "A pure theory of local expenditures", Journal of Political Economy, vol. 24, n 3, October, pp. 416-424.

TORRES E., 1998, Le cadre de vie urbain : essai d'une économie de la qualité. Faculté des sciences économiques et sociales, Université des sciences et technologies de Lille I : 386.

THIEBAUT L., 1992, Demandes de biens d'environnement et interventions publiques en agriculture- cas de la France, sciences économiques, Université de Montpellier, Unité de formation et de recherche de droit et des sciences économiques, Montpellier, $350 \mathrm{p}$.

VIGOUROUX I., 1999, Asymétries informationnelles et marché politique, Sciences économiques, Paris I - Panthéon Sorbonne - UFR d'économie, Paris, 311p. 


\section{NOTES}

1. Nous ne retenons que les biens collectifs environnementaux et excluons de notre champ les services publics de proximité (école, poste...) ou les services aux personnes (soins à domicile...).

2. Nous ne pouvons pas affirmer que les conflits d'usage sont plus nombreux aujourd'hui qu'hier, nous savons qu'ils sont différents eu égard à l'évolution de la société, même s'ils représentent parfois une version actualisée d'antagonismes du passé.

3. Individus censés être atteints du syndrome NIMBY (Not In My BackYard : pas dans ma cour).

4. Nous rejoignons sur ce point BENARD (1985, p. 42) : " (...) le concept d'externalité, excluant les ajustements marchands spontanés, (il) a été (selon l'auteur) réservé aux phénomènes d'externalité technologique".

5. la distinction entre encombrement et surexploitation provient seulement du décalage dans le temps de la réception de l'effet externe négatif.

6. www.airpur.org

\section{RÉSUMÉS}

Les conflits d'usage révèlent la dynamique contemporaine des fonctions de l'espace rural et notamment la montée en puissance de la fonction cadre de vie de la campagne et de sa confrontation avec les autres usages du rural (productif et de réservation). La thèse défendue dans cet article consiste à affirmer que les conflits d'usage de nombreux biens collectifs environnementaux localisés constituent une procédure d'ajustement de la décision publique les concernant, compte tenu des limites de l'expression des préférences des usagers par la procédure du vote lors des échéances électorales, et de la difficulté à fuir les nuisances (stratégie de l'exit). L'analyse de cinq années de contentieux du Tribunal Administratif de Clermont-Ferrand (1998 à 2002) pour le département du Puy de Dôme, et le traitement d'articles de presse parus entre 1999 et 2002 dans le quotidien régional La Montagne donnent un éclairage de la diversité des conflits, des moyens de droit convoqués par les adversaires, et de la nature des solutions matérielles apportées aux différends.

Land-uses and neighbourhood conflicts reveal the rise of the rural living environment and with its confrontation with the other functions of the countryside (productive and "nature"). The thesis claimed in this article consists in defending the idea that land-uses conflicts mean as a deal with public decision-makers, taking into account the limits of the expression of the preferences by the voting procedure, and when exit is difficult. The focus is put on a French administrative territory: Puy de Dôme. The five year analysis (1998 to 2002) of litigation of the administrative court of Clermont-Ferrand, and the treatment by a local daily press La Montagne from 1999 to 2002, offer an explanation of different ways of right convened by opponents and the main ways to the material solutions brought to the disagreements. 
INDEX

Mots-clés : biens publics locaux, cadre de vie, conflits d'usage, décision publique, effet externe négatif, espace rural et périurbain

Keywords : land-uses and neighbourhood conflicts, local public goods, negative external effect, public decision-making, rural living environment

\section{AUTEUR}

\section{PHILIPPE JEANNEAUX}

UMR METAFORT Clermont-Ferrand - UMR CESAER Dijon, jeanneaux@enitac.fr. Enseignant chercheur contractuel à l'ENITAC de Clermont-Ferrand. Doctorant en économie à l'UMR CESAER Dijon, sous la direction de Philippe PERRIER-CORNET. Le travail de thèse porte sur l'analyse économique des conflits d'usage dans les espaces ruraux et périurbains. Dernières communications : AZNAR, O., GUERIN, M. JEANNEAUX P. et al. (2005) Conflits d'usage et services environnementaux dans les espaces ruraux : Éléments pour l'analyse économique des politiques publiques environnementales. Symposium international Territoires et enjeu du développement régional, Lyon. 12 p. JEANNEAUX, P., KIRAT, T., (2005) Observer les conflits d'usage : que nous apprend le contentieux judiciaire et administratif sur le développement des régions ? Symposium international Territoires et enjeu du développement régional Lyon. 18 p. 